\title{
Obesity Is Associated With Increased Basal and Postprandial $\beta$-Cell Insulin Secretion Even in the Absence of Insulin Resistance
}

\author{
Stephan van Vliet, ${ }^{1,2}$ Han-Chow E. Koh, ${ }^{1}$ Bruce W. Patterson, ${ }^{1}$ Mihoko Yoshino, ${ }^{1}$ Richard LaForest, ${ }^{3}$ \\ Robert J. Gropler, ${ }^{3}$ Samuel Klein, ${ }^{1}$ and Bettina Mittendorfer ${ }^{1}$
}

Diabetes 2020;69:2112-2119 | https://doi.org/10.2337/db20-0377

\begin{abstract}
We tested the hypothesis that obesity, independent of insulin resistance, is associated with increased insulin secretion. We compared insulin kinetics before and after glucose ingestion in lean healthy people and people with obesity who were matched on multiorgan insulin sensitivity (inhibition of adipose tissue lipolysis and glucose production and stimulation of muscle glucose uptake) as assessed by using a two-stage hyperinsulinemiceuglycemic pancreatic clamp procedure in conjunction with glucose and palmitate tracer infusions and positron emission tomography. We also evaluated the effect of diet-induced weight loss on insulin secretion in people with obesity who did not improve insulin sensitivity despite marked ( $20 \%)$ weight loss. Basal and postprandial insulin secretion rates were $>\mathbf{5 0} \%$ greater in people with obesity than lean people even though insulin sensitivity was not different between groups. Weight loss in people with obesity decreased insulin secretion by $35 \%$ even though insulin sensitivity did not change. These results demonstrate that increased insulin secretion in people with obesity is associated with excess adiposity itself and is not simply a compensatory response to insulin resistance. These findings have important implications regarding the pathogenesis of diabetes because hyperinsulinemia causes insulin resistance and insulin hypersecretion is an independent risk factor for developing diabetes.
\end{abstract}

Obesity is associated with increased plasma insulin concentrations during basal conditions and postprandially. It has been proposed that the increase in plasma insulin in people with obesity is an adaptive response, caused by both increased insulin secretion and decreased insulin clearance, to compensate for impaired insulin sensitivity $(1,2)$. However, it has also been proposed that increased $\beta$-cell insulin secretion in people with obesity is a primary abnormality that precedes, and contributes to, the development of insulin resistance $(3,4)$. This notion is supported by the findings that both basal and glucose-stimulated insulin secretion are greater in isolated pancreata from obese than from lean animals $(5,6)$. Moreover, an experimentally induced increase in plasma insulin causes insulin resistance in lean healthy people $(7,8)$. A better understanding of the relationship between obesity and insulin kinetics (secretion and removal) has important clinical implications because there is a direct relationship between insulin secretion and plasma insulin concentration and the risk of developing prediabetes and type 2 diabetes $(9,10)$.

The purpose of the present studies was to test the hypothesis that obesity, independent of insulin resistance, is associated with increased insulin secretion during basal conditions (after an overnight fast) and after glucose ingestion. Accordingly, we evaluated insulin kinetics both during basal conditions and after glucose ingestion in lean healthy people and people with obesity who were matched on insulin sensitivity with lean participants. Multiorgan insulin sensitivity (ability of insulin to inhibit lipolysis of adipose tissue triglycerides,
${ }^{1}$ Center for Human Nutrition, Washington University School of Medicine, St. Louis, MO

2Duke Molecular Physiology Institute, Duke University, Durham, NC ${ }^{3}$ Mallinckrodt Institute of Radiology at Washington University School of Medicine, St. Louis, M0

Corresponding author: Bettina Mittendorfer, mittendb@wustl.edu

Received 13 April 2020 and accepted 1 July 2020
Clinical trial reg. nos. NCT02994459, NCT03408613, NCT02207777, and NCT01299519, clinicaltrials.gov

This article contains supplementary material online at https://doi.org/10.2337/ figshare.12601082

(C) 2020 by the American Diabetes Association. Readers may use this article as long as the work is properly cited, the use is educational and not for profit, and the work is not altered. More information is available at https://www.diabetesjournals .org/content/license. 
suppress hepatic glucose production, and stimulate muscle glucose uptake) was assessed by using a two-stage hyperinsulinemic-euglycemic pancreatic clamp procedure in conjunction with stable isotopically labeled glucose and palmitate tracer infusions, ${ }^{18} \mathrm{~F}$-fluorodeoxyglucose $\left(\left[{ }^{18} \mathrm{~F}\right] \mathrm{FDG}\right)$ injection, and positron emission tomography (PET). In addition, we evaluated the effect of weight loss on insulin secretion in people with obesity who did not improve whole-body insulin sensitivity (glucose infusion rate during a hyperinsulinemic-euglycemic clamp procedure) despite marked weight loss to determine whether a decrease in body fat mass, independent of an improvement in insulin sensitivity, reduces insulin secretion.

\section{RESEARCH DESIGN AND METHODS}

\section{Study Participants}

\section{Study 1 (Lean and Insulin-Sensitive Obese)}

The data reported here were obtained from 16 men and women (8 lean [3 men, 5 women; 28-56 years old] and 8 with obesity [ 3 men, 5 women; $30-58$ years old]) selected from a pool of participants who completed a rigorous assessment of metabolic function as part of other studies (NCT02994459 and NCT03408613, ClinicalTrials.gov). The inclusion criteria for this study were 1) BMI $\geq 18.5$ and $<25.0 \mathrm{~kg} / \mathrm{m}^{2}$ (lean group) or $\geq 30.0$ and $<45.0 \mathrm{~kg} / \mathrm{m}^{2}$ (obese group), 2) no evidence of diabetes or other chronic illness, 3) normal fasting plasma glucose $(<100 \mathrm{mg} / \mathrm{dL})$ and glucose tolerance (plasma glucose $<140 \mathrm{mg} / \mathrm{dL} 2 \mathrm{~h}$ after ingesting 75 g glucose), 4) no use of medications that could affect the study outcomes, 5) no use of tobacco products or excessive consumption of alcohol (more than one drink a day), and 6) not involved in structured exercise for $\geq 1.5 \mathrm{~h}$ per week. The participants in the lean and obese groups were specifically chosen to ensure matching between groups on sex, fasting plasma glucose concentration, and insulin sensitivity (assessed as insulin-stimulated muscle glucose uptake).

\section{Study 2 (People With Obesity Before and After Weight Loss)}

The data reported here were obtained from six women $\left(46 \pm 5\right.$ years old) with obesity $\left(\mathrm{BMI} \geq 30.0 \mathrm{~kg} / \mathrm{m}^{2}\right)$ who participated in studies that evaluated the effects of weight loss on insulin sensitivity and insulin secretion (NCT02207777 and NCT01299519, ClinicalTrials.gov). They were specifically chosen because they were the only participants who achieved at least a $15 \%$ weight loss (induced by dietary calorie restriction) who did not improve whole-body insulin sensitivity (as assessed as glucose infusion rate during a hyperinsulinemiceuglycemic clamp procedure). Additional inclusion criteria for this study were 1) no evidence of diabetes or other chronic illness, 2) no use of medications that could affect the study outcomes, 3) no use of tobacco products or excessive consumption of alcohol (more than one drink a day), and 4) not involved in structured exercise for $\geq 1.5 \mathrm{~h}$ per week.
Written informed consent was obtained from all participants before initiating the study protocols. The protocols were approved by the Human Research Protection Office at Washington University.

\section{Body Composition Analysis and Metabolic Testing \\ Study 1 (Lean and Insulin-Sensitive Obese)}

Each participant completed the following assessments: 1) detailed body composition analyses, 2) insulin kinetics before and after ingesting an oral glucose load, 3) basal glucose and palmitate kinetics determined by using intravenous $\left[6,6-{ }^{2} \mathrm{H}_{2}\right]$ glucose and $\left[\mathrm{U}-{ }^{13} \mathrm{C}\right]$ palmitate infusions, and 4) effects of insulin on endogenous glucose production, adipose tissue fatty acid release, and muscle glucose uptake determined by using a two-stage hyperinsulinemic-euglycemic pancreatic clamp procedure in conjunction with $\left[6,6-{ }^{2} \mathrm{H}_{2}\right]$ glucose and $\left[\mathrm{U}-{ }^{13} \mathrm{C}\right]$ palmitate infusions and $\left[{ }^{18} \mathrm{~F}\right] \mathrm{FDG}$ injection and PET.

\section{Study 2 (People With Obesity Before and After Weight Loss)}

Each participant completed the following assessments before and after weight loss: 1) detailed body composition analyses, 2) insulin kinetics before and after ingesting an oral glucose load, and 3) whole-body insulin sensitivity determined by using a hyperinsulinemic-euglycemic clamp procedure.

Detailed descriptions of the testing procedures and sample collection and analysis protocols are provided in the Supplementary Material.

\section{Calculations}

Palmitate rate of appearance $\left(R_{a}\right)$ in plasma was calculated by dividing the $\left[\mathrm{U}-{ }^{13} \mathrm{C}\right]$ palmitate infusion rate by the average plasma palmitate tracer-to-tracee ratio (11). Glucose $\mathrm{R}_{a}$ in plasma was calculated by dividing the $\left[{ }^{2} \mathrm{H}_{2}\right]$ glucose infusion rate by the average plasma glucose tracerto-tracee ratio (12). During basal conditions, this value represents the endogenous glucose production rate; during the hyperinsulinemic clamp procedure, the amount of glucose that was infused was subtracted from this value to obtain the endogenous glucose production rate (12). Fractional glucose uptake rate $\left(R_{g}\right)$ in the erector spinae and oblique muscles were calculated by using Patlak graphical analysis of the tissue and arterial blood-specific activity time curves; absolute rates of muscle glucose uptake ( $\mu \mathrm{mol} /$ $\mathrm{kg}$ muscle/min) were then derived by dividing the product of fractional $\mathrm{R}_{\mathrm{g}}$ and plasma glucose concentration by 1.2 (lumped constant) (13). Values from the two muscles were averaged for presentation. Insulin secretion rate was determined by fitting the plasma $C$-peptide concentrations to a two-compartment model using a time-variable insulin secretion rate (14). Insulin clearance rate (volume of plasma cleared of insulin per unit of time) was calculated by dividing the insulin secretion rate (basal secretion rate and secretion rate area under the curve [AUC] after glucose ingestion, respectively) by the plasma insulin concentration (basal concentration and concentration AUC after glucose ingestion, respectively); after glucose ingestion, this amount was 
Table 1-Body composition in lean healthy people and people with obesity

\begin{tabular}{lcc} 
& Lean $(n=8)$ & Obese $(n=8)$ \\
\hline $\mathrm{BMI}\left(\mathrm{kg} / \mathrm{m}^{2}\right)$ & $23(2)$ & $35(4)^{\star}$ \\
\hline Body mass $(\mathrm{kg})$ & $64(5)$ & $100(14)^{\star}$ \\
\hline Fat-free mass $(\mathrm{kg})$ & $45(7)$ & $57(11)^{\star}$ \\
\hline Body fat $(\%$ of total body mass) & $30(8)$ & $43(10)^{\star}$ \\
\hline Fat mass $(\mathrm{kg})$ & $19(6)$ & $44(13)^{\star}$ \\
\hline Subcutaneous abdominal adipose tissue volume $\left(\mathrm{cm}^{3}\right)$ & $2,503(1,128)$ & $9,167(3,589)^{\star}$ \\
\hline Intra-abdominal adipose tissue volume $\left(\mathrm{cm}^{3}\right)$ & $1,196(618)$ & $3,099(984)^{\star}$ \\
\hline Intrahepatic triglyceride content $(\%)$ & $1.8(0.9)$ & $2.8(1.9)$ \\
\hline Data are mean (SD). ${ }^{*} P<0.05$ vs. lean. & &
\end{tabular}

corrected for the amount of insulin that was not cleared during $2 \mathrm{~h}$ and therefore accumulated in plasma.

\section{Statistical Analysis}

All data sets were tested for normality by using the KolmogorovSmirnov test; skewed data sets were log-transformed before analysis. A two-sample $t$ test for independent groups was used to compare outcomes in the lean and obese groups. A two-sample $t$ test for paired samples was used to compare outcomes before and after weight loss. Values are reported as mean $\pm \mathrm{SD}$ or median (IQR) unless otherwise noted. $P \leq$ 0.05 was considered statistically significant.

\section{Sample Size Estimation}

The primary study outcomes of insulin secretion rate during basal conditions and after glucose ingestion are usually $\geq 45 \%$ greater in people with obesity than in those who are lean (15). On the basis of the interindividual variability of insulin secretion after glucose ingestion we observed previously (16), we estimated that eight participants per group would be needed to detect at least a $45 \%$ difference in our lean and obese groups with a power of 0.8 and an $\alpha$ of 0.05 . Six participants would be needed to detect a $\geq 30 \%$ weight loss-induced change in insulin secretion.
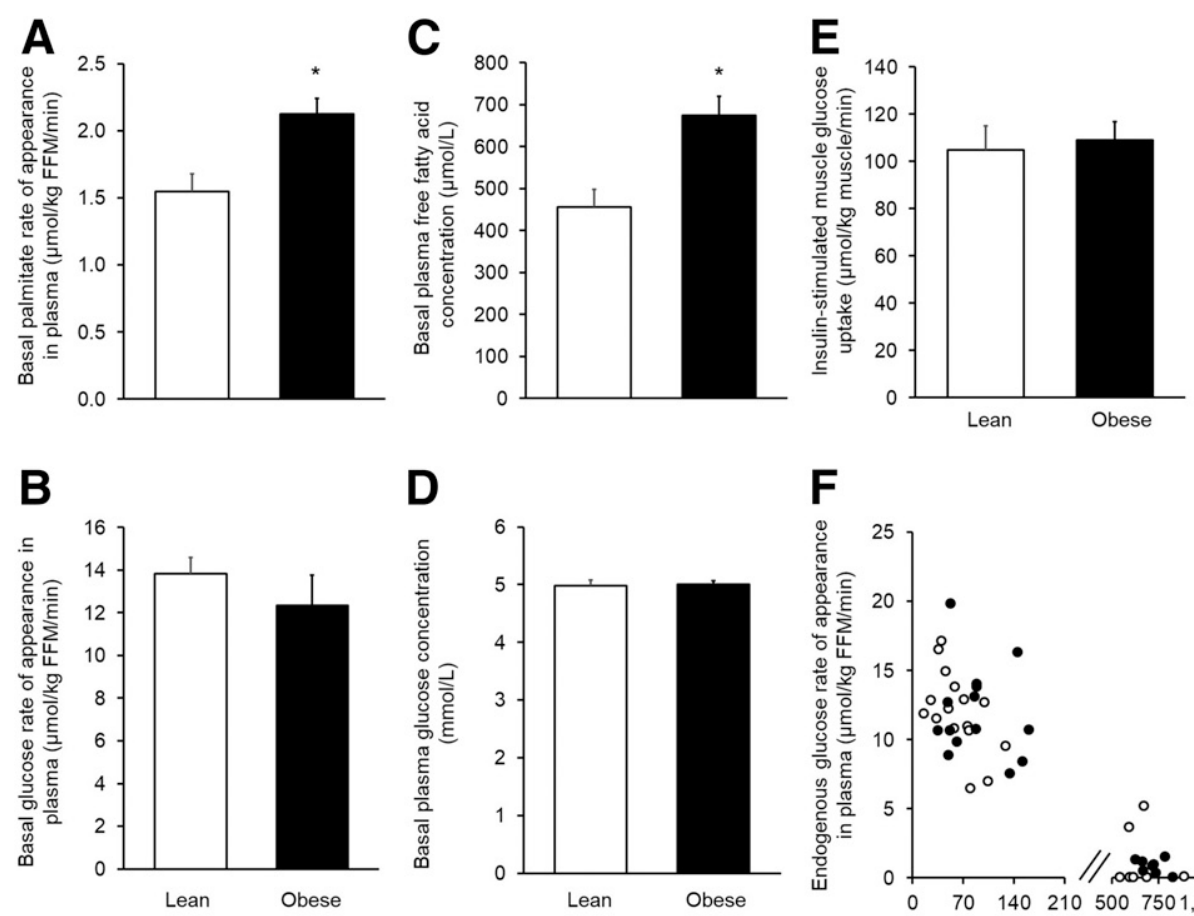
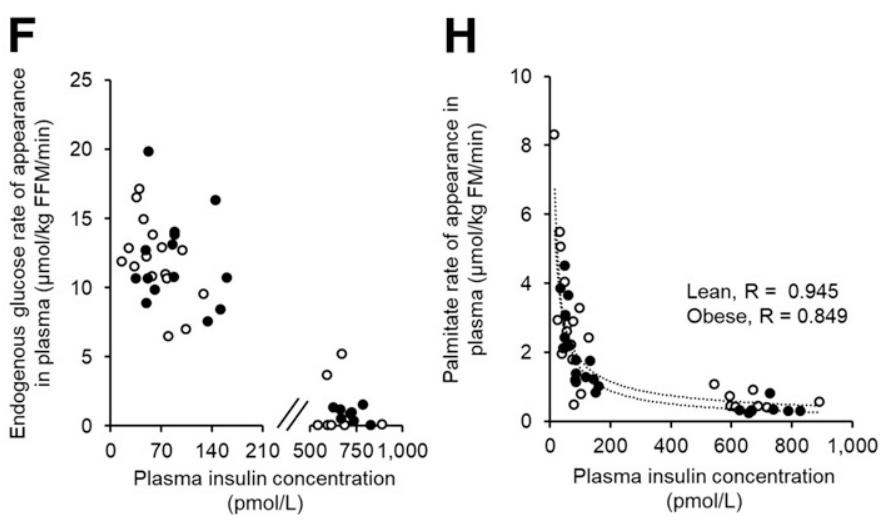

Figure 1-Basal palmitate and glucose $\mathrm{R}_{\mathrm{a}}$ in plasma $(A$ and $B)$ and plasma free fatty acid and glucose concentrations $(C$ and $D)$, insulinstimulated muscle $R_{g}$ determined by PET after intravenous injection of $\left[{ }^{18} \mathrm{~F}\right] \mathrm{FDG}(E)$, and the relationships between plasma insulin concentration during basal conditions and during the hyperinsulinemic-euglycemic clamp and endogenous glucose $R_{a}$ in plasma relative to fat-free mass (FFM) $(F)$, total palmitate $R_{a}$ in plasma $(G)$, and palmitate $R_{a}$ in plasma relative to fat mass $(H)$ in the lean $(n=8)$ and obese $(n=8)$ groups. Data in panels $A-E$ are mean \pm SEM. ${ }^{*} P<0.05$ vs. the lean group. FFM, fat-free mass. 

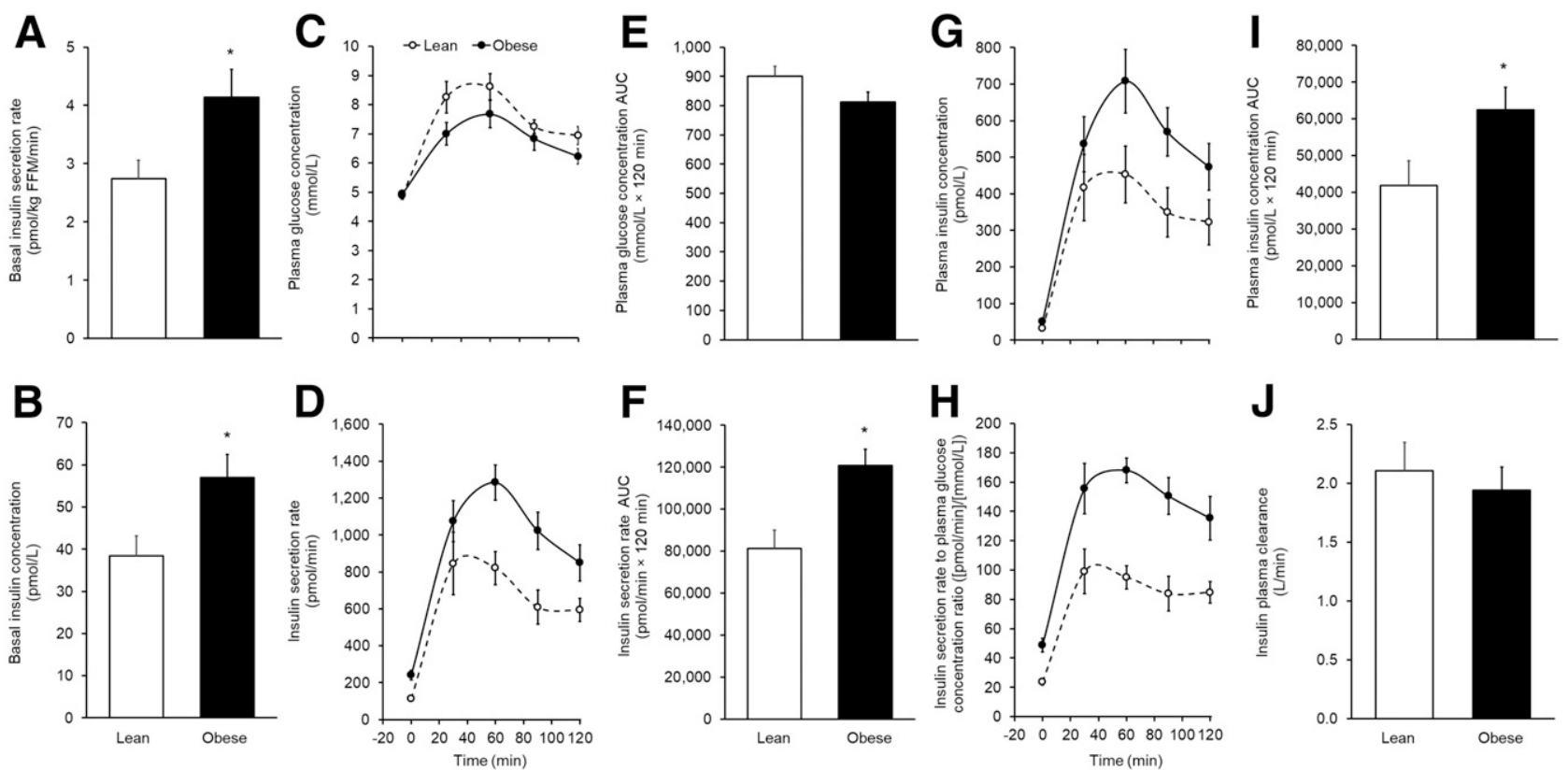

Figure 2-Basal insulin secretion rate $(A)$ and concentration $(B)$ and plasma glucose and insulin concentrations and insulin kinetics before and after glucose ingestion $(C-J)$ in the lean $(n=8)$ and obese $(n=8)$ groups. Data are mean \pm SEM. ${ }^{*} P<0.05$ vs. the lean group. FFM, fatfree mass.

\section{Data and Resource Availability}

The data generated and/or analyzed during the current study are available from the corresponding author upon reasonable request. No resources were generated.

\section{RESULTS}

\section{Study 1 (Lean and Insulin-Sensitive Obese) \\ Body Composition}

Fat-free mass was $\sim 25 \%$ greater in the obese than the lean group, and total body fat mass and abdominal subcutaneous and intra-abdominal adipose tissue volumes were more than double in the obese compared with the lean group (Table 1). Intrahepatic triglyceride content was not different between the lean and obese groups (Table 1).

\section{Basal Fatty Acid and Glucose Kinetics and Concentrations}

Total basal palmitate $R_{a}$ in plasma (expressed as $\mu \mathrm{mol} /$ min) and palmitate $R_{a}$ in relation to fat-free mass and body surface area were greater in the obese than the lean group (Fig. $1 A$ and Supplementary Table 1 ), whereas palmitate $\mathrm{R}_{\mathrm{a}}$ in relation to fat mass (Supplementary Table 1) was not different between groups. Total basal endogenous glucose $R_{a}\left(\mu \mathrm{mol} / \mathrm{min}\right.$ ) and endogenous glucose $R_{a}$ in relation to fat-free mass and body surface area (Fig. $1 B$ and Supplementary Table 1) were not different between the obese and lean groups. Basal fatty acid concentration in plasma (Fig. $1 C$ ) was $\sim 50 \%$ greater in the obese than the lean group, whereas basal plasma glucose concentration (Fig. 1D) was matched a priori and therefore not different between the groups.

\section{Multiorgan Insulin Sensitivity}

Insulin-stimulated muscle glucose uptake was matched a priori and therefore not different between the lean and obese groups (Fig. 1E). Endogenous glucose $\mathrm{R}_{\mathrm{a}}$ in plasma was inversely related to both plasma insulin concentration and total insulin delivery (i.e., insulin secretion rate during basal conditions, sum of insulin secretion rate and intravenous insulin infusion rate during the clamp procedure) in both the lean and the obese groups, without differences between the groups (Fig. $1 \mathrm{~F}$ and Supplementary Fig. 1). Total palmitate $R_{a}$ in plasma was inversely related to plasma insulin concentration in both the lean and the obese groups and greater in the obese than the lean group at any given plasma insulin concentration (Fig. $1 G$ ). Palmitate $\mathrm{R}_{\mathrm{a}}$ in plasma relative to fat-free mass was also greater in the obese than the lean group at any given plasma insulin concentration (Supplementary Fig. 1), but palmitate $R_{a}$ in plasma relative to fat mass was not different between the groups (Fig. 1H).

\section{Basal Insulin Kinetics and Concentration}

Basal plasma C-peptide concentration (Supplementary Table 2), insulin secretion rate (total and relative to fat-free mass and body surface area), and plasma insulin concentration (Fig. $2 A$ and $B$ and Supplementary Table 1 ) were $\sim 50 \%$ greater in the obese than the lean group. Insulin clearance rate (volume of plasma cleared of insulin per minute) was not different between the obese and lean groups (Supplementary Table 2). 

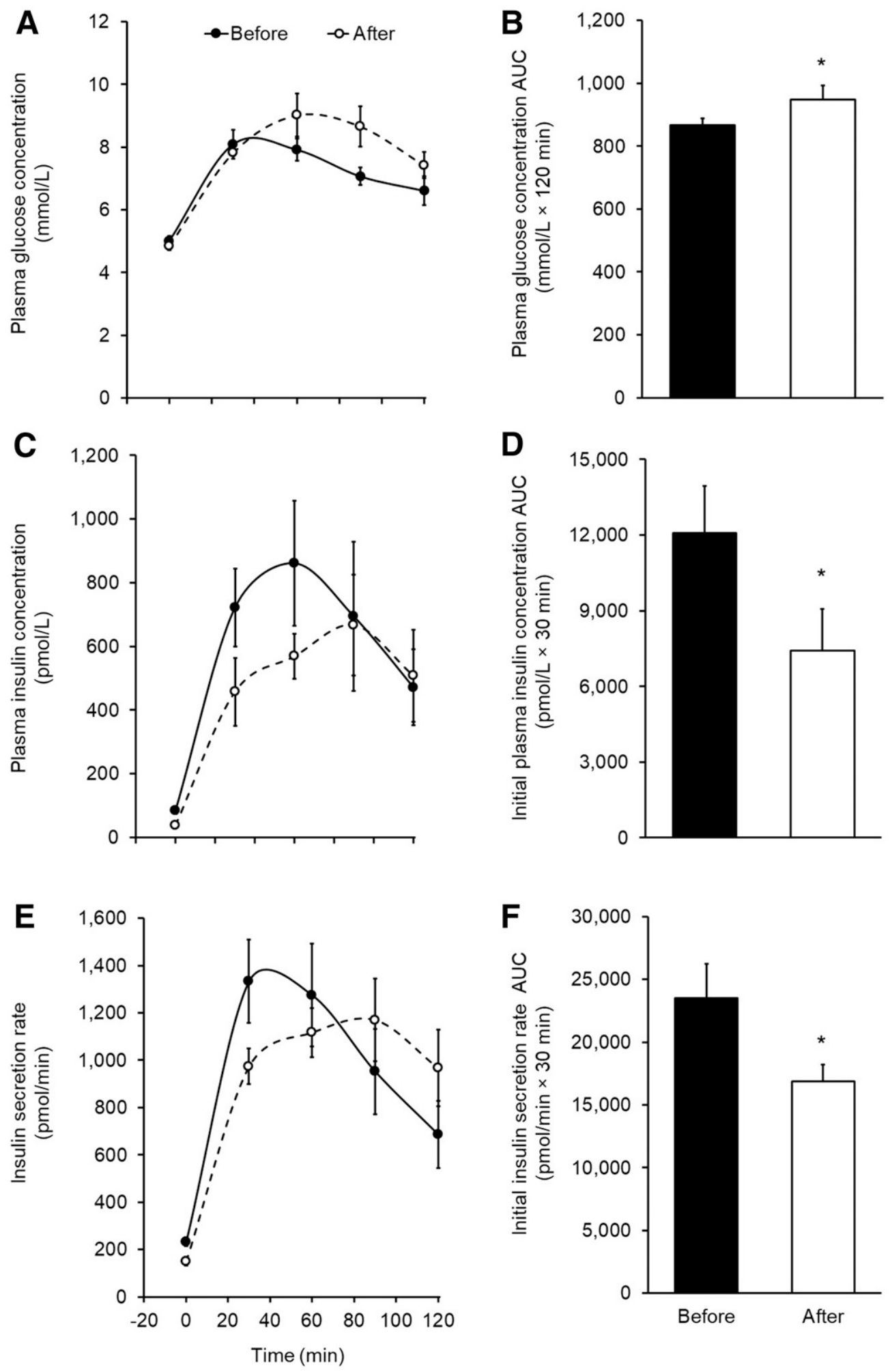

Figure 3-Effect of diet-induced weight loss on plasma glucose and insulin concentrations $(A-D)$ and insulin secretion rate $(E$ and $F)$ before and after glucose ingestion in women with obesity $(n=6)$ who did not improve whole-body insulin sensitivity despite marked $(\sim 20 \%)$ weight loss. Data are mean \pm SEM. ${ }^{\star} P<0.05$ vs. before weight loss. 


\section{Metabolic Response to Glucose Ingestion}

The plasma glucose concentration AUC after glucose ingestion (Fig. $2 C$ and $E$ ) tended to be lower in the obese than the lean group, but the difference did not reach statistical significance $(P=0.09)$. The plasma insulin concentration AUC after glucose ingestion was greater in the obese than the lean group (Fig. 2D and F). Basal and postprandial plasma glucose-dependent insulinotropic polypeptide and glucagon-like peptide 1 concentrations were not different between the obese and lean groups (Supplementary Fig. 2). Plasma glucagon concentration was $\sim 15 \%$ greater in the obese than the lean group during basal conditions and after glucose ingestion, but the differences were not statistically significant (Supplementary Fig. 2). Plasma free fatty acid concentration was almost completely suppressed within the first hour after glucose ingestion in both the lean and the obese groups, without a difference between the groups (data not shown). The insulin secretion rate was $50-100 \%$ greater in the obese than the lean group throughout the 120-min time period after glucose ingestion (Fig. $2 G$ and $I$ ). The increase in insulin secretion after glucose ingestion (incremental AUC above basal values) was also greater in the obese than the lean group (92 \pm 22 vs. $68 \pm 25 \mathrm{nmol}$ during the 2 -h time period after glucose ingestion, $P=0.057$ ) even though the amount of glucose ingested relative to body size was less in the obese than the lean group (1.4 \pm 0.3 vs. $1.7 \pm 0.2 \mathrm{~g} / \mathrm{kg}$ fat-free mass or $36 \pm 3$ vs. $43 \pm 3 \mathrm{~g} / \mathrm{m}^{2}$ body surface area, both $P<0.05$ ) and the increase in plasma glucose concentration after glucose ingestion (incremental AUC above basal values) tended to be lower in the obese than the lean group $(220 \pm 91$ vs. $315 \pm$ $41 \mathrm{mmol} / \mathrm{L} \times 120 \mathrm{~min}$, respectively, $P=0.09$ ). Both the insulin secretion rate-to-plasma glucose concentration ratio (Fig. $2 \mathrm{H}$ ) and the plasma insulin concentration-to-glucose concentration ratio (data not shown) were $\sim 80 \%$ greater in the obese than the lean group. Insulin clearance rate (volume of plasma cleared of insulin per minute) was not different between the lean and obese groups (Fig. $2 J$ ), and the total amount of insulin cleared from plasma over $120 \mathrm{~min}$ after glucose ingestion was greater in the obese than the lean group (115 \pm 21 and $79 \pm 24 \mathrm{nmol}$, respectively, $P<0.01)$.

\section{Study 2 (People With Obesity Before and After Weight Loss)}

Body Composition and Whole-Body Insulin Sensitivity

On average, participants lost $19 \pm 2 \%$ of their body weight and $30 \pm 4 \%$ of their fat mass (Supplementary Table 4 ). The total glucose infusion rate $(\mu \mathrm{mol} / \mathrm{min})$ needed to maintain euglycemia $(5.8 \pm 0.1$ and $5.7 \pm 0.2 \mathrm{mmol} / \mathrm{L}$ before and after weight loss, respectively) during the hyperinsulinemic clamp procedure was less after than before weight loss (Supplementary Table 4), whereas the glucose infusion rate relative to fat-free mass was not different before and after weight loss $(0.11 \pm 0.02$ and $0.09 \pm 0.02[\mu \mathrm{mol} / \mathrm{kg}$ fat-free $\mathrm{mass} / \mathrm{min}] /[\mathrm{pmol}$ insulin/L]). Before weight loss, people with obesity in this study were very similar to participants with obesity in study 1 with regard to body composition (Table 1) and whole-body insulin sensitivity (glucose infusion rate during high-dose insulin infusion $0.10 \pm 0.02[\mu \mathrm{mol} / \mathrm{kg}$ fat-free $\mathrm{mass} / \mathrm{min}] /[\mathrm{pmol}$ insulin/L]).

\section{Glucose, Fatty Acid, and Insulin Concentrations and Insulin Secretion}

Basal total plasma fatty acid concentration was $\sim 20 \%$ lower $(474[462 ; 1,196]$ vs. $608[550 ; 2,151] \mu \mathrm{mol} / \mathrm{L})$ and basal insulin secretion rate and concentration were $\sim 35 \%$ lower (all $P<0.05$ ) after than before weight loss (Fig. 3), whereas basal plasma glucose concentration was not affected by weight loss (Fig. 3). During the first $30 \mathrm{~min}$ after glucose ingestion, plasma glucose concentration was not different before and after weight loss, but the total plasma glucose concentration AUC after glucose ingestion was greater after than before weight loss (Fig. 3), presumably because the same total amount of glucose, and therefore more glucose relative to body size, was ingested after than before weight loss. In contrast, insulin secretion and concentration during the first $30 \mathrm{~min}$ of glucose ingestion were $\sim 30 \%$ lower after than before weight loss (Fig. 3), and total insulin secretion and concentration AUCs after glucose ingestion were not different before and after weight loss.

\section{DISCUSSION}

We integrated the results from different research techniques, including a two-stage hyperinsulinemic-euglycemic pancreatic clamp procedure in conjunction with stable isotopically labeled glucose and palmitate tracer infusions and $\left[{ }^{18} \mathrm{~F}\right] \mathrm{FDG}$ injection and PET, an oral glucose tolerance test, and mathematical modeling, to determine the effect of obesity, independent of multiorgan insulin resistance, on insulin kinetics before and after glucose ingestion. To isolate the effects of obesity from insulin resistance, we matched our lean and obese groups on insulin sensitivity. Therefore, our obese group represents a subset of people with obesity who are considered metabolically healthy (17). We found that the insulin secretion rates during basal conditions (when plasma glucose concentrations were the same in the obese and lean groups) and after glucose ingestion (when plasma glucose concentration tended to be lower in the obese than the lean group) were greater in the obese than the lean group. In addition, the insulin clearance rate (volume of plasma cleared of insulin per minute) was not different between the lean and obese groups. Accordingly, the high plasma insulin concentration in the obese group was due to increased insulin secretion, not decreased insulin removal. We also evaluated the effect of diet-induced weight loss on insulin secretion before and after glucose ingestion in women with obesity who did not improve whole-body insulin sensitivity (assessed as glucose infusion rate during a hyperinsulinemic-euglycemic clamp procedure) despite $\sim 20 \%$ body weight loss and an $\sim 30 \%$ decrease in fat mass. Weight loss decreased basal and early postprandial insulin secretion rates even though insulin sensitivity did not change and the early postprandial increase in 
plasma glucose was not different after than before weight loss. The data from our studies, therefore, challenge the notion that increased insulin secretion in people with obesity is simply a compensatory mechanism to increase plasma insulin to overcome insulin resistance $(1,2)$. Instead, our data demonstrate that $\beta$-cell insulin secretion is increased in people with obesity, even in the absence of insulin resistance.

The mechanisms responsible for greater insulin secretion in our participants with obesity are unclear. It has been proposed that overconsumption and chronic overstimulation of $\beta$-cells with insulinogenic nutrients results in $\beta$-cell hyperplasia, which in turn causes $\beta$-cell hyperreactivity to glucose $(3,6,18)$. It is also possible that increased fatty acid delivery from adipose tissue to pancreatic $\beta$-cells in the obese group caused insulin hypersecretion by accelerating glucose catabolism in $\beta$-cells and through lipid signaling $(3,6,18)$. Moreover, basal insulin hypersecretion itself could have been involved in the greater insulin response to glucose ingestion in participants with obesity because insulin augments glucose-stimulated insulin secretion $(19,20)$ by increasing $\beta$-cell $\mathrm{Ca}^{2+}$ signaling and glucose catabolism (21-23).

It has been proposed that chronic hyperinsulinemia results in downregulation of insulin receptors and postreceptor defects in insulin signaling and, therefore, causes insulin resistance and increases the risk for prediabetes and type 2 diabetes $(3,4,7,8)$. However, the high insulin secretion rate and plasma insulin concentrations were not associated with insulin resistance in our participants with obesity. Moreover, the marked decrease in insulin secretion that we observed after weight loss in our participants was not associated with improved insulin sensitivity. It is unlikely that the magnitude or duration of hyperinsulinemia in our participants is insufficient to cause insulin resistance because even a moderate $(\sim 20 \%)$ experimentally induced increase in plasma insulin concentration markedly impairs insulin action within $24 \mathrm{~h}$ (8). Even though insulin resistance is considered the primary factor involved in the pathogenesis of metabolic abnormalities associated with obesity (17), a chronic high demand for insulin secretion can cause $\beta$-cell stunning (i.e., inability to appropriately sense insulin secretagogues after years of overstimulation) and eventual $\beta$-cell exhaustion and type 2 diabetes $(3,24)$. Therefore, our data provide a potential mechanism for the increased risk of developing type 2 diabetes in people with obesity who are metabolically healthy (17) and suggest that weight loss might reduce the risk for type 2 diabetes in people with obesity by decreasing insulin secretion, even if insulin sensitivity does not improve.

The data from our study do not support the notion that increased fatty acid release from adipose tissue in people with obesity always causes insulin resistance in muscle and liver $(3,6,18,25-27)$. Although adipose tissue insulin sensitivity, assessed as lipolytic rate relative to fat mass, was not different between the lean and obese groups, basal lipolytic rate relative to fat-free mass was much greater in the obese than the lean group because of the marked increase in the ratio of fat mass to fat-free mass in the obese compared with the lean group $(0.81 \pm 0.32$ and $0.45 \pm 0.15$, respectively, $P=0.01$ ). However, muscle and liver insulin sensitivity were not different between the obese and lean groups. These findings suggest that participants in our obese group may have been protected from the adverse metabolic effects of fatty acids on insulin action possibly because of alterations in cellular fatty acid uptake and metabolism in metabolically healthy compared with metabolically unhealthy obesity. It is also possible that the integrated daily plasma free fatty acid "load" was not much different in the lean and obese groups because of the marked postprandial suppression of lipolysis and plasma fatty acid concentration.

Our findings have important implications for the interpretation of commonly used indices that rely on plasma glucose and insulin concentrations to assess insulin resistance in people with obesity, such as HOMA of insulin resistance, Matsuda composite insulin sensitivity, and oral glucose insulin sensitivity (28). These approaches assume that plasma glucose concentration is primarily a direct result of insulin action in liver (glucose production) and muscle (glucose disposal) and do not take into account insulin-independent mechanisms (e.g., glucose uptake by non-insulin-sensitive vital organs and tissues, glucosemediated suppression of glucose production and stimulation of muscle glucose uptake) that regulate basal and postprandial plasma glucose concentrations $(29,30)$. In fact, on the basis of these indices, our participants would have been incorrectly characterized as being insulin resistant (Supplementary Table 3).

In summary, we found that increased insulin secretion during basal conditions and after glucose ingestion occurs even in the absence of insulin resistance in people with obesity. Furthermore, marked weight loss decreases insulin secretion both during basal conditions and after glucose ingestion, even in people who do not demonstrate an improvement in insulin sensitivity. The mechanisms responsible for the obesity-associated increase in insulin secretion are unclear but likely involve $\beta$-cell hyperreactivity to glucose because of overconsumption-induced $\beta$-cell hyperplasia and altered $\beta$-cell glucose catabolism and lipid signaling because of both increased fatty acid delivery from adipose tissue and basal insulin secretion. The obesity-associated increase in $\beta$-cell insulin secretion has important clinical implications because increased insulin secretion is an independent risk factor for prediabetes and type 2 diabetes, most likely because the chronic high demand for insulin secretion results in $\beta$-cell exhaustion. Accordingly, interventions for preventing type 2 diabetes in people with obesity not only should focus on insulin resistance but also include treatments that can "rest" $\beta$-cells.

Acknowledgments. The authors thank the staff of the Center for Human Nutrition, the Clinical Translational Research Unit, the Clinical Translational Imaging Unit, and the Division of Radiological Sciences for help with participant 
recruitment, scheduling, and testing and technical assistance with sample processing and data analysis and the study subjects for participation.

Funding. The work presented in this article was supported by Center for Scientific Review grants DK-115400, DK-101578, DK-56341 (Nutrition Obesity Research Center), DK-020579 (Diabetes Research Center), and UL1-TR-000448 (Clinical Translational Science Award) and grants from the American Diabetes Association (1-18-ICTS-119) and the Pershing Square Foundation.

Duality of Interest. No potential conflicts of interest relevant to this article were reported.

Author Contributions. S.v.V., H.-C.E.K., B.W.P., M.Y., R.L., R.J.G., S.K., and B.M. contributed to the acquisition, analysis, and interpretation of the data and to the revision of the manuscript for important intellectual content. R.J.G. and B.M. designed the study. B.M. wrote the first draft of the report. B.M. is the guarantor of this work and, as such, had full access to all the data in the study and takes responsibility for the integrity of the data and the accuracy of the data analysis.

\section{References}

1. Kahn SE, Prigeon RL, McCulloch DK, et al. Quantification of the relationship between insulin sensitivity and beta-cell function in human subjects. Evidence for a hyperbolic function. Diabetes 1993;42:1663-1672

2. Bergman RN, Ader M, Huecking K, Van Citters G. Accurate assessment of betacell function: the hyperbolic correction. Diabetes 2002;51(Suppl. 1):S212-S220

3. Erion K, Corkey BE. $\beta$-Cell failure or $\beta$-cell abuse? Front Endocrinol (Lausanne) 2018:9:532

4. Shanik MH, Xu Y, Skrha J, Dankner R, Zick Y, Roth J. Insulin resistance and hyperinsulinemia: is hyperinsulinemia the cart or the horse? Diabetes Care 2008; 31(Suppl. 2):S262-S268

5. Curry DL, Stern JS. Dynamics of insulin hypersecretion by obese Zucker rats. Metabolism 1985;34:791-796

6. Unger RH. Lipotoxicity in the pathogenesis of obesity-dependent NIDDM. Genetic and clinical implications. Diabetes 1995;44:863-870

7. Rizza RA, Mandarino LJ, Genest J, Baker BA, Gerich JE. Production of insulin resistance by hyperinsulinaemia in man. Diabetologia 1985;28:70-75

8. Iozzo P, Pratipanawatr T, Pijl H, et al. Physiological hyperinsulinemia impairs insulin-stimulated glycogen synthase activity and glycogen synthesis. Am J Physiol Endocrinol Metab 2001;280:E712-E719

9. Aston-Mourney K, Proietto J, Morahan G, Andrikopoulos S. Too much of a good thing: why it is bad to stimulate the beta cell to secrete insulin. Diabetologia 2008; $51: 540-545$

10. Tricò $D$, Natali $A$, Arslanian $S$, Mari $A$, Ferrannini E. Identification, pathophysiology, and clinical implications of primary insulin hypersecretion in nondiabetic adults and adolescents. JCI Insight 2018;3:e124912

11. Mittendorfer B, Liem O, Patterson BW, Miles JM, Klein S. What does the measurement of whole-body fatty acid rate of appearance in plasma by using a fatty acid tracer really mean? Diabetes 2003;52:1641-1648

12. Smith Gl, Yoshino J, Kelly SC, et al. High-protein intake during weight loss therapy eliminates the weight-loss-induced improvement in insulin action in obese postmenopausal women. Cell Rep 2016;17:849-861
13. Kelley DE, Williams KV, Price JC, Goodpaster B. Determination of the lumped constant for $\left[{ }^{18} \mathrm{~F}\right.$ fluorodeoxyglucose in human skeletal muscle. J Nucl Med 1999; 40:1798-1804

14. Van Cauter E, Mestrez F, Sturis J, Polonsky KS. Estimation of insulin secretion rates from C-peptide levels. Comparison of individual and standard kinetic parameters for C-peptide clearance. Diabetes 1992;41:368-377

15. Ferrannini E, Gastaldelli A, Miyazaki Y, Matsuda M, Mari A, DeFronzo RA. beta-Cell function in subjects spanning the range from normal glucose tolerance to overt diabetes: a new analysis. J Clin Endocrinol Metab 2005;90:493-500

16. Weiss EP, Albert SG, Reeds DN, et al. Calorie restriction and matched weight loss from exercise: independent and additive effects on glucoregulation and the incretin system in overweight women and men. Diabetes Care 2015;38:12531262

17. Smith Gl, Mittendorfer B, Klein S. Metabolically healthy obesity: facts and fantasies. J Clin Invest 2019;129:3978-3989

18. Prentki M, Corkey BE, Murthy Madiraju SR. Lipid-associated metabolic signalling networks in pancreatic beta cell function. Diabetologia 2020;63:10-20

19. Halperin F, Lopez X, Manning R, Kahn CR, Kulkarni RN, Goldfine AB. Insulin augmentation of glucose-stimulated insulin secretion is impaired in insulinresistant humans. Diabetes 2012;61:301-309

20. Weir GC. Glucolipotoxicity, $\beta$-cells, and diabetes: the emperor has no clothes. Diabetes 2020;69:273-278

21. Aspinwall CA, Qian WJ, Roper MG, Kulkarni RN, Kahn CR, Kennedy RT. Roles of insulin receptor substrate-1, phosphatidylinositol 3-kinase, and release of intracellular $\mathrm{Ca} 2+$ stores in insulin-stimulated insulin secretion in beta -cells. $\mathrm{J}$ Biol Chem 2000;275:22331-22338

22. Assmann A, Ueki K, Winnay JN, Kadowaki T, Kulkarni RN. Glucose effects on beta-cell growth and survival require activation of insulin receptors and insulin receptor substrate 2. Mol Cell Biol 2009;29:3219-3228

23. Leibiger B, Wahlander K, Berggren PO, Leibiger IB. Glucose-stimulated insulin biosynthesis depends on insulin-stimulated insulin gene transcription. $J$ Biol Chem 2000;275:30153-30156

24. Ferrannini E. The stunned beta cell: a brief history. Cell Metab 2010;11:349352

25. Bergman RN. Non-esterified fatty acids and the liver: why is insulin secreted into the portal vein? Diabetologia 2000;43:946-952

26. Petersen MC, Shulman Gl. Mechanisms of insulin action and insulin resistance. Physiol Rev 2018;98:2133-2223

27. Ghaben AL, Scherer PE. Adipogenesis and metabolic health. Nat Rev Mol Cell Biol 2019;20:242-258

28. Muniyappa R, Lee S, Chen H, Quon MJ. Current approaches for assessing insulin sensitivity and resistance in vivo: advantages, limitations, and appropriate usage. Am J Physiol Endocrinol Metab 2008;294:E15-E26

29. Dube S, Errazuriz-Cruzat I, Basu A, Basu R. The forgotten role of glucose effectiveness in the regulation of glucose tolerance. Curr Diab Rep 2015;15:605 30. Alatrach M, Agyin C, Mehta R, Adams J, DeFronzo RA, Abdul-Ghani M. Glucose-mediated glucose disposal at baseline insulin is impaired in IFG. J Clin Endocrinol Metab 2019;104:163-171 